\title{
Hypertension Canada's 2017 guidelines for diagnosis, risk assessment, prevention and treatment of hypertension in adults for pharmacists: An update
}

Sarah A. Lamb, BSP; Yazid N. Al Hamarneh, BSc(Pharm), PhD;

Sherilyn K. D. Houle, BSP, PhD; Alexander A. Leung, MD, MPH;

Ross T. Tsuyuki, BSc(Pharm), PharmD, MSc, FCSHP, FACC, FCAHS

Hypertension CANAdA (Formerly THE CANAdian Hypertension Education Program, CHEP) has been publishing evidence-based guidelines for the diagnosis and management of hypertension since 1999. ${ }^{1}$ The 2017 guidelines, ${ }^{2}$ released earlier this year, denote the 19th annual synthesis and update of evidence to date in hypertension. These guidelines, together with knowledge translation programs for health care professionals, ${ }^{3}$ have helped to improve the levels of detection and management of hypertension in Canada and reduced associated cardiovascular mortality. ${ }^{4,5}$ Indeed, the proportion of patients with controlled blood pressure in Canada has significantly increased from $13.2 \%$ in $1992^{5}$ to $68.1 \%$ in 2012-2013. ${ }^{4}$

A multidisciplinary expert panel is responsible for the synthesis and dissemination of Hypertension Canada's guidelines. To date, the panel has consisted of physicians, nurses and pharmacists, among other disciplines.

Pharmacists are well positioned to manage hypertension, as they see patients with chronic conditions more frequently than any other health care professional. ${ }^{6}$ Furthermore, the growing body of evidence supporting pharmacist interventions is indisputable. ${ }^{7-13}$ As such, Hypertension Canada has recognized pharmacists' expanded scope of practice as one approach to improve the detection and management of hypertension in Canada.

The Canadian Pharmacists Journal has regularly published pharmacist-specific hypertension guidelines, with the most recent full set of guidelines published in $2011^{14}$ and regular updates thereafter. This article highlights updates that have been integrated into the current 2017 Hypertension Canada guidelines, in addition to elements that the authors feel are still important from previous publications. Readers requiring Hypertension Canada's full guidelines are encouraged to refer to the Canadian Journal of Cardiology, ${ }^{2}$ www.hypertension.ca or previous versions of pharmacist-specific publications in the Canadian Pharmacists Journal.

\section{What's new}

The 2017 Hypertension Canada guidelines present 6 changes relevant to pharmacists (Box 1).

\section{Indications for drug therapy in adults with hypertension}

The previous guideline recommendations for alternate blood pressure targets in the frail and elderly have been removed.

Antihypertensive therapy should be provided to all patients without macrovascular target
(C) The Author(s) 2017 DOl:10.1177/1715163517743525 
Box 1 Changes to 2017 Hypertension Canada guidelines relevant to pharmacists

1. Alternate blood pressure targets in the frail and elderly have been removed.

2. Longer-acting thiazide-like diuretics are preferred over traditional, short-acting thiazide diuretics.

3. Single-pill combinations are recommended as first-line options for the treatment of hypertension.

4. When lowering systolic blood pressure (SBP) in patients with coronary artery disease (especially with isolated systolic hypertension), use caution when diastolic blood pressure (DBP) is $\leq 60 \mathrm{mmHg}$, especially in patients with left ventricular hypertrophy.

5. Avoid lowering SBP to $<140 \mathrm{mmHg}$ in the hyperacute phase (first 24 hours) of intracerebral hemorrhage.

6. Pharmacists are advised to remain vigilant when managing and monitoring patients with hypertension, since resistant hypertension was identified as an area of concern in the 2017 guidelines.

organ damage, such as myocardial infarction and stroke, or other cardiovascular risk factors (e.g., dyslipidemia, smoking, diabetes mellitus, sedentary lifestyle and obesity) with average diastolic blood pressure (DBP) readings of $\geq 100 \mathrm{mmHg}$ (Grade A) and/or average systolic blood pressure $(\mathrm{SBP})$ readings of $\geq 160 \mathrm{mmHg}$ (Grade A). ${ }^{15}$ In addition, antihypertensive therapy should be strongly considered for all patients with macrovascular target organ damage or other independent cardiovascular risk factors with average DBP readings of $\geq 90 \mathrm{mmHg}$ (Grade A) and/or average SBP readings of $\geq 140 \mathrm{mmHg}$ (Grade B for $140-160 \mathrm{mmHg}$, Grade A for $>160 \mathrm{mmHg}$ ). All patients should be treated to achieve a SBP target of $<140 \mathrm{mmHg}$ (Grade C) and/or a DBP target of $<90 \mathrm{mmHg}$ (Grade A); however, caution should be used in elderly patients experiencing orthostasis.

A post hoc analysis of the HYVET trial ${ }^{16}$ and a subgroup analysis of the SPRINT trial ${ }^{17,18} \mathrm{dem}$ onstrated that blood pressure lowering in elderly patients (age $\geq 80$ years and $\geq 75$ years in HYVET and SPRINT, respectively) significantly reduced the incidence of major cardiovascular events (hazard ratio [HR], 0.66; 95\% confidence interval $[\mathrm{CI}], 0.51-0.85),{ }^{17}$ mortality (HR, $0.67 ; 95 \%$ CI, $0.49-0.91)^{17}$ and stroke (HR, 0.64; 95\% CI, $0.42-0.96),{ }^{16}$ regardless of age or baseline frailty. There was no difference in the rates of serious adverse events, however, rates of renal dysfunction were significantly increased in the intensive treatment group in SPRINT. ${ }^{17}$ Moreover, individuals with dementia, limited life expectancy and those institutionalized were excluded from the HYVET and SPRINT trials, limiting generalizability to these groups. These findings are supported by a meta-analysis conducted by Xie and colleagues, ${ }^{19}$ which demonstrated a favourable relationship between intensive blood pressure lowering and reduced major cardiovascular events in older adults ( $\geq 62$ years) (relative risk [RR], 0.81; 95\% CI, 0.69-0.96).

\section{Choice of therapy for adults with hypertension without compelling indications for specific agents}

Long-acting, thiazide-like diuretics

(chlorthalidone and indapamide) are preferred to thiazide diuretics for the initial management of hypertension (Grade B), although both thiazide and thiazide-like diuretics remain first-line options.

This preference is guided by evidence supporting the use of long-acting diuretics to reduce blood pressure and cardiovascular events. ${ }^{20-22} \mathrm{~A}$ meta-analysis conducted by Olde Engberink and colleagues $^{20}$ showed that after adjusting for differences in blood pressure reduction, thiazidelike diuretics reduced the risk of cardiovascular events and heart failure by an additional $12 \%(p$ $=0.049)$ and $21 \%(p=0.023)$, respectively, compared to thiazide diuretics. Moreover, only thiazide-like diuretics reduced the risk of coronary events (RR, 0.76; CI, 0.61-0.96; $I^{2}=0 \%$ ) and allcause mortality (RR, 0.84; CI, 0.74-0.96; $I^{2}=0 \%$ ) compared to placebo. ${ }^{20}$ In addition, Roush and colleagues $^{21}$ and Pareek and colleagues ${ }^{22}$ demonstrated a greater reduction in blood pressure 
with extended-release hydrochlorothiazide and thiazide-like diuretics compared to conventional hydrochlorothiazide.

Single-pill combinations (SPCs) are recommended as an initial treatment option (Grade A) based on evidence supporting their effectiveness in reducing blood pressure and cardiovascular events. ${ }^{23-27}$ In addition, SPCs have been shown to improve adherence and reduce the incidence of adverse events. ${ }^{28,29}$

Combination antihypertensive therapy is associated with a reduced likelihood of cardiovascular events compared to monotherapy. ${ }^{23,30}$ Furthermore, SPCs have been shown to be more effective at reducing blood pressure compared to monotherapy. A meta-analysis of 42 randomized clinical trials demonstrated that the combination of 2 drugs of different classes resulted in a 5-fold greater reduction in systolic blood pressure compared to doubling the dose of $1 \mathrm{drug}^{24}$ These findings are supported by evidence from the STITCH study, ${ }^{25}$ where a larger blood pressure reduction $(-5.2 /-2.2)$ was observed in individuals receiving initial fixed-dose combination therapy of an angiotensin-converting enzyme (ACE) inhibitor or angiotensin-receptor blocker (ARB) and diuretic, compared to monotherapy with uptitration at 6 months. ${ }^{25}$ Moreover, a greater percentage of individuals reached their target blood pressure $(64.7 \%$ vs $52.7 \%, p=0.03){ }^{25}$

A meta-analysis conducted by Sherrill and colleagues ${ }^{28}$ found that the use of SPCs was associated with improved adherence rates and lower health care costs compared to the use of the individual components. Furthermore, another meta-analysis conducted by Law and colleages ${ }^{29}$ found that fixed-dose antihypertensive combinations were associated with a lower incidence of adverse events and improved efficacy.

When choosing an SPC, it is recommended to use an ACE inhibitor combined with a calcium channel blocker (CCB) (Grade A), an ARB combined with a CCB (Grade B) or an ACE or ARB combined with a diuretic (Grade B). This recommendation is supported by the ACCOMPLISH trial, ${ }^{26}$ which randomized high-risk adults to either benazepril plus amlodipine or benazepril plus hydrochlorothiazide. The composite of cardiovascular death and major adverse cardiovascular events was significantly reduced with benazepril plus amlodipine compared to benazepril plus hydrochlorothiazide (HR, 0.80; $95 \%$ CI, 0.72-0.90). The HOPE- $3^{27}$ and STITCH ${ }^{25}$ trials support the use of an ACE inhibitor or ARB combined with a diuretic. While the HOPE-3 trial reported no significant difference in the 2 coprimary outcomes for individuals with an intermediate risk of cardiovascular events with candesartan-hydrochlorothiazide vs placebo overall, a significant reduction in the coprimary endpoints was demonstrated in those with higher blood pressure or hypertension $(\mathrm{SBP}>143.5 \mathrm{mmHg}){ }^{27}$

\section{Treatment of hypertension in association with ischemic heart disease}

When treating SBP to target in patients with established coronary artery disease (CAD) (especially in those with isolated systolic hypertension), exercise caution when DBP is $\leq 60 \mathrm{mmHg}$ due to concerns with exacerbating myocardial ischemia, especially for patients with left ventricular hypertrophy (Grade D).

This recommendation is supported by post hoc analyses of several studies showing that in patients with $\mathrm{CAD}$, reducing blood pressure below a specific threshold was associated with an increased risk of coronary events. ${ }^{31-34}$ Individuals with left ventricular hypertrophy may be at greatest risk due to increased myocardial demand and decreased coronary perfusion during diastole. Indeed, an association between reduced coronary blood flow and increased left ventricular mass was noted in patients with $\mathrm{CAD}$ and was most pronounced at DBP $<70$ mmHg. ${ }^{35}$ These findings are in line with a meta-analysis conducted by Rabkin. ${ }^{36}$

Even so, the 2017 guidelines $^{2}$ still strongly recommend antihypertensive therapy for most patients who can tolerate blood pressure reduction, especially those with moderate or severe hypertension, due to the benefits of blood pressure reduction in these high-risk individuals, which likely outweigh the associated risks.

\section{Treatment of hypertension in association with hemorrhagic stroke}

In patients in the hyperacute phase (first 24 hours) of intracerebral hemorrhage, avoid SBP lowering to $<140 \mathrm{mmHg}$ due to a lack of benefit (relative to a target of $<180 \mathrm{mmHg}$ ) and the potential for harm (Grade A). 
Trial evidence demonstrated no benefit and potential harm with SBP targets of $<140$ $\mathrm{mmHg}$ in the acute phase of intracerebral hemorrhage. $^{37,38}$ INTERACT-2 randomized 2839 patients who had a spontaneous intracerebral hemorrhage within 6 hours of onset to a target SBP of either $<140 \mathrm{mmHg}$ or $<180 \mathrm{mmHg}$ (applied within the first hour of spontaneous intracerebral hemorrhage presentation). ${ }^{37}$ There was no statistical difference in the primary outcome of death or stroke-related disability at 90 days when comparing the 2 SBP targets (odds ratio [OR], 0.87; 95\% CI, 0.75-1.01). These findings are consistent with the ATACH-2 trial, ${ }^{38}$ where 1000 patients presenting with spontaneous intracerebral hemorrhage were randomized to SBP targets of 110 to $139 \mathrm{mmHg}$ or 140 to $179 \mathrm{mmHg}$. Treatment was initiated within 4.5 hours after symptom onset and continued for the next 24 hours. ${ }^{38}$ No difference in the primary outcome of death or stroke-related disability was observed between the 2 arms; however, there was a trend toward greater adverse events in the lower SBP arm. ${ }^{38}$

\section{Resistant hypertension}

The 2017 guidelines recognize resistant hypertension as an area of concern; however, due to a lack of event-related outcomes from the PATHWAY-2 trial, $^{39}$ no specific guideline recommendations were made.

Resistant hypertension affects $10 \%$ to $20 \%$ of patients treated for high blood pressure and is associated with increased cardiovascular risk compared to other forms of hypertension. ${ }^{40,41}$ It is defined as uncontrolled blood pressure despite the use of 3 or more antihypertensive agents from different classes (including a diuretic), or hypertension that is controlled with 4 or more agents. ${ }^{40,41}$ Optimal treatment strategies for this heterogeneous disorder remain uncertain. While the PATHWAY-2 trial showed that spironolactone was the most effective add-on drug in lowering SBP compared to doxazosin, bisoprolol and placebo $(-12.8 \mathrm{mmHg}[-13.8$ to -11.8$]$ for spironolactone, $-8.7 \mathrm{mmHg}[-9.7$ to -7.7$]$ for doxazosin, $-8.3 \mathrm{mmHg}$ [ -9.3 to -7.3 ] for bisoprolol and $-4.1 \mathrm{mmHg}(-5.1$ to -3.1 ] for placebo), this trial did not assess cardiovascular outcomes. ${ }^{39}$ As such, no specific guideline recommendations were generated on the basis of its results.

\section{What is still important}

\section{Diagnosis and accurate measurement of blood} pressure

Using standardized measurements and validated equipment (Grade D), trained health care professionals, including pharmacists, should assess blood pressure in all patients at appropriate visits to monitor antihypertensive therapy and evaluate cardiovascular risk (Grade D). Pharmacists should ensure they only use and sell validated blood pressure monitors. Validation means that instruments have been tested against a gold standard. Electronic (oscillometric) upper arm devices should be used preferentially due to inaccuracies seen with manual (auscultatory) blood pressure measurements (Grade C). ${ }^{42}$

Figure 1 illustrates the recommended diagnostic algorithm for hypertension. After an initial finding of in-office elevated blood pressure, it is recommended to use out-of-office blood pressure measurements to confirm the initial diagnosis of hypertension, as it better predicts cardiovascular outcomes compared to in-office measurements. ${ }^{43}$ Moreover, out-of-office blood pressure measurements can identify both white coat and masked hypertension. ${ }^{43}$

Ambulatory blood pressure monitoring is the preferred out-of-office measurement (Grade D) as it provides a greater number of readings, as well as information regarding nocturnal blood pressure, and is considered a strong predictor of cardiovascular events. ${ }^{44}$ When using this method, patients can be diagnosed with hypertension if the mean ambulatory 24-hour blood pressure is $\geq 130 / 80 \mathrm{mmHg}$ or the mean awake ambulatory blood pressure is $\geq 135 / 85 \mathrm{mmHg}$ (Grade C). If ambulatory blood pressure monitoring is not available, cannot be tolerated or patient preference precludes use, home blood pressure monitoring can be used (Grade D). A diagnosis of hypertension can be made if the mean home blood pressure is $\geq 135 / 85 \mathrm{mmHg}$ following duplicate measures, morning and evening, for 7 days, with the first day's values excluded from the overall mean calculation (Grade C).

Automated office blood pressure (AOBP) is the preferred method for in-office measurements (Grade D), with a mean blood pressure of $\geq 135 / 85 \mathrm{mmHg}$ considered elevated (Grade D).

If the patients' in-office blood pressure is elevated (AOBP $\geq 135 / 85 \mathrm{mmHg}$ ) and the mean awake home blood pressure is $<135 / 85 \mathrm{mmHg}$ 


\section{FIGURE 1 Hypertension diagnostic algorithm}

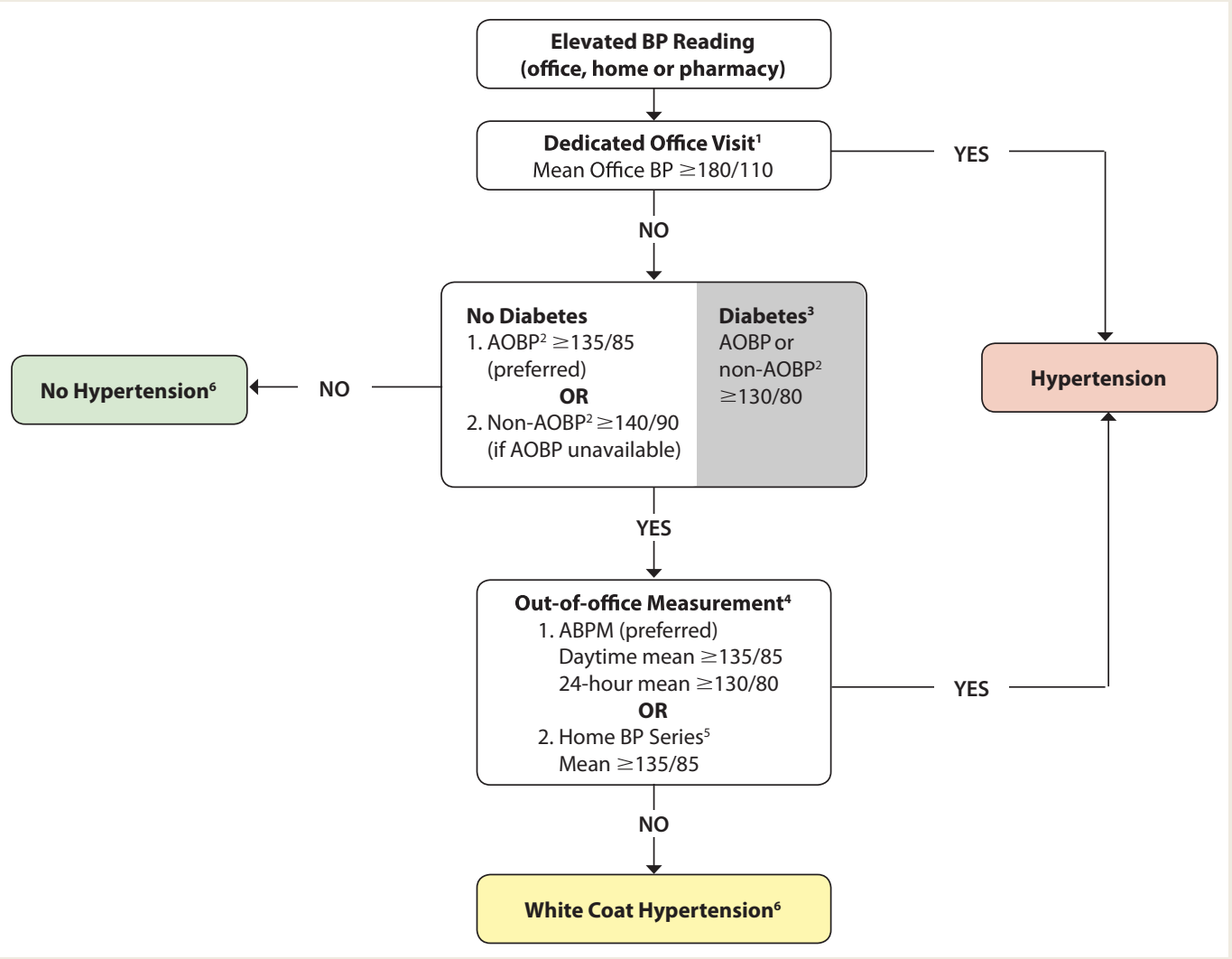

ABPM, ambulatory blood pressure measurement; $A O B P$, automated office blood pressure; BP, blood pressure.

${ }^{1}$ If $A O B P$ is used, use the mean calculated and displayed by the device. If non-AOBP is used, take at least 3 readings, discard the first, and calculate the mean of the remaining measurements. A history and physical exam should be performed and diagnostic tests ordered. ${ }^{2} \mathrm{AOBP}$ is performed with the patient unattended in a private area. Non-AOBP is performed using an electronic upper arm device with the provider in the room.

${ }^{3}$ Diagnostic thresholds for AOBP, ABPM and home BP in patients with diabetes have yet to be established (and might be lower than $130 / 80 \mathrm{mmHg}$ ).

${ }^{4}$ Serial office measurements over 3 to 5 visits can be used if ABPM or home measurement is not available.

${ }^{5}$ For a home BP series, 2 readings are taken each morning and evening for 7 days ( 28 total). Discard the first day readings and average the last 6 days.

${ }^{6}$ Annual BP measurement is recommended to detect progression to hypertension.

Adapted with permission from Leung AA, Daskalopoulou SS, Dasgupta K, et al. Hypertension Canada's 2017 guidelines for diagnosis, risk assessment, prevention and treatment of hypertension in adults. Can J Cardiol 2017;33(5):557-76.

or the mean 24-hour ambulatory blood pressure is $<130 / 80 \mathrm{mmHg}$, then a diagnosis of white coat hypertension can be made (Grade D). Antihypertensive therapy should not be initiated under these circumstances (Grade C) due to a lack of benefit and increased risk of adverse events.

\section{Assessment and management of overall} cardiovascular risk and vascular protection High blood pressure represents a major risk factor for premature death and disability. ${ }^{45,46}$ Furthermore, $80 \%$ of patients with hypertension have at least 1 additional risk factor for cardiovascular disease,${ }^{47}$ further increasing the likelihood of an event. As such, overall cardiovascular risk should be assessed in all patients with hypertension.

Cardiovascular risk engines can be used to more accurately predict the patient's overall cardiovascular risk (Grade A) and improve the efficiency of antihypertensive therapy (Grade D). Pharmacists may consider counselling patients about their overall cardiovascular risk and consider using comparative risk analogies, such as "cardiovascular age," "heart age" or "vascular age" (Grade B) to help advise patients about their risk. This approach improves both the 
effectiveness of risk factor modification as well as the patient's perception of risk. ${ }^{48-52}$

The $\mathrm{R}_{\mathrm{x}} \mathrm{EACH}$ study randomized 723 patients to receive pharmacist intervention (including prescribing) or usual care. ${ }^{13}$ Pharmacist intervention comprised individualized cardiovascular risk assessment and education regarding this risk. ${ }^{13}$ Compared to usual care, greater reductions in cardiovascular risk $(21 \%, p<0.001)$ and blood pressure $(-9.37 /-2.92 \mathrm{mmHg}, p<0.001)$ were observed in the pharmacist intervention group at 3 months. ${ }^{13}$ These findings highlight the importance of overall cardiovascular risk assessment in this patient population.

Statin therapy is recommended in hypertensive patients who have $\geq 3$ cardiovascular risk factors (Grade A, in patients $>40$ years) or established atherosclerotic disease (Grade A, irrespective of age). Risk factors include the following:

- Male sex

- Age $\geq 55$ years

- Left ventricular hypertrophy

- Electrocardiogram (ECG) abnormalities

- Peripheral arterial disease

- Previous stroke or transient ischemic attack

- Albuminuria or proteinuria

- Diabetes

- Tobacco use

- Family history of premature cardiovascular disease

- Total cholesterol to high-density lipoprotein cholesterol ratio $\geq 6$

Low-dose acetylsalicylic acid (ASA) therapy may also be considered for patients with hypertension who are 50 years or older (Grade B); however, caution should be used in patients whose blood pressure remains uncontrolled (Grade C).

Pharmacists should assess tobacco use regularly and advise on tobacco cessation in all patients (Grade C). Tobacco use represents a significant contributor to elevated blood pressure and cardiovascular risk. ${ }^{53}$ As such, tobacco cessation is crucial to reducing vascular risk. The pharmacists' role in smoking cessation is supported by a meta-analysis conducted by Saba and colleagues. ${ }^{54}$ Five randomized controlled trials with a total of 1426 smokers were included in the analysis. ${ }^{54}$ Compared with control groups, pharmacist interventions demonstrated greater abstinence rates (RR, 2.21; 95\% CI, 1.49-3.29), including longterm abstinence (RR, 2.40; 95\% CI, 1.37-4.23). ${ }^{54}$
Pharmacist interventions included providing advice and counselling to patients in either a one-on-one or a group setting. Pharmacologic therapy (nicotine replacement) was also provided in one study. ${ }^{54}$ Pharmacists' interventions should include offering patients both advice and pharmacotherapy (e.g., nicotine replacement therapy, varenicline or bupropion) with a goal of tobacco cessation. Stead and colleagues ${ }^{55}$ demonstrated that patients were more likely to have sustained smoking cessation when given brief advice on quitting vs usual care (5.8\% vs $3.7 \%$ ). A Cochrane network meta-analysis ${ }^{56}$ reported an even greater likelihood of quitting and sustained tobacco cessation with a combination of advice and pharmacotherapy compared to advice plus placebo.

Intensive blood pressure management to a SBP target of $\leq 120 \mathrm{mmHg}$ may be considered in select high-risk patients who are 50 years or older and whose blood pressure is $\geq 130 \mathrm{mmHg}$ (Grade B). This recommendation stems from the SPRINT trial, ${ }^{18}$ which demonstrated a reduction in cardiovascular events secondary to intensive blood pressure lowering (SBP $<120 \mathrm{mmHg}$ ). High-risk patients are defined as those with the following ${ }^{18}$ :

- Clinical or subclinical cardiovascular disease

- Chronic kidney disease (nondiabetic nephropathy, proteinuria <1 g/d, estimated glomerular filtration rate [eGFR] $20-59 \mathrm{~mL} / \mathrm{min} / 1.73 \mathrm{~m}^{2}$ )

- Estimated 10-year cardiovascular risk $\geq 15 \%$ (Framingham Risk Score)

- Age $\geq 75$ years

Pharmacists should ensure patient risk assessment is consistent with the above criteria before initiating intensive treatment. In addition, patients should consent to receiving intensive treatment that includes multiple antihypertensive medications and an increased frequency of follow-up visits. A careful risk-benefit analysis should be performed due to the propensity for adverse events with intensive blood pressure management. Moreover, such treatment should be avoided in certain high-risk groups (Grade B) due to a lack of supporting evidence. These highrisk groups include the following:

- Patients with heart failure (ejection fraction $<35 \%$ ) or recent myocardial infarction (within the past 3 months)

- An indication for, but not currently receiving, a beta-blocker 
- Frail or institutionalized elderly individuals

- Diabetes mellitus

- Previous stroke

- eGFR $<20 \mathrm{~mL} / \mathrm{min} / 1.73 \mathrm{~m}^{2}$

- Unwilling or unable to adhere to multiple medications

- Standing SBP $<110 \mathrm{mmHg}$

- Inability to measure SBP accurately

- Known secondary cause(s) of hypertension

\section{Adherence}

Patient nonadherence to antihypertensive therapy is common and has a significant impact on blood pressure control and cardiovascular complications. ${ }^{57-59}$ Early discontinuation of treatment is one of the most common causes of nonadherence. ${ }^{60}$ One systematic review and meta-analysis found that $45 \%$ of patients with hypertension were not taking their antihypertensive therapy, and a higher proportion $(83.7 \%)$ of patients with uncontrolled hypertension were nonadherent to therapy. ${ }^{57}$ Thus, evaluating and assisting patients with adherence is a crucial step in the management of hypertension. Health care providers can improve patient adherence using a comprehensive approach addressing factors relating to patients, medications and themselves. ${ }^{58,59}$ Moreover, pharmacist intervention and management of blood pressure has been shown to improve adherence rates. ${ }^{61}$

- To address patient factors:

- Educate patients, family members and caregivers about the condition and associated treatments

- Encourage patients to monitor their blood pressure regularly at home

- To address medication factors:

- Encourage the use of once-daily regimens, single-pill combination and unit-of-dose packaging to simplify the treatment regimen

- Tailor the patient's medication-taking schedule to fit his or her daily routine

- To address health care provider factors:

- Assess adherence to therapy at every patient encounter

- Use a multidisciplinary team approach, including work-site health care providers, to improve adherence

\section{Evidence for pharmacist-led interventions}

The pharmacists' role in chronic disease management is becoming increasingly prominent as a result of an expanded scope of practice and the growing strain on the health care system. Research has demonstrated the benefits of pharmacist-led interventions for hypertension management. ${ }^{7-12}$ Indeed, Hypertension Canada is exceedingly supportive of the pharmacist's expanded role to improve hypertension detection and management, as well as reduce the risk of cardiovascular events.

A systematic review and meta-analysis of 39 randomized controlled trials comprising over 14,000 patients showed that pharmacists' interventions-namely, patient education, recommendations to physicians and medication management-resulted in greater reductions of both SBP $(-7.6 \mathrm{mmHg}$; $95 \% \mathrm{CI},-9$ to -6.3 ) and DBP (-3.9 mmHg; 95\% CI, -5.1 to -2.8 ) compared with usual care. ${ }^{9}$ Interestingly, the most pronounced effect on blood pressure reduction was seen when the pharmacist led the intervention, resulting in a SBP reduction of $-8.5 \mathrm{mmHg}$ and a DBP reduction of -4.6 mmHg. Results from the R ACTION trial demonstrated an even greater effect on blood pressure reduction when pharmacist interventions included independent prescribing for hypertension (mean SBP reduction $18.3 \mathrm{mmHg}$, adjusted difference $6.6 \mathrm{mmHg}) .{ }^{10} \mathrm{In}$ addition, patients in the pharmacist prescribing arm were 2.3 times more likely to achieve target blood pressures. A recent economic analysis evaluating the costeffectiveness of pharmacist care for managing hypertension found that pharmacist-led interventions were economically dominant, being more effective (i.e., reduced blood pressure) and with a lifetime cost savings of $\$ 6364 .{ }^{62}$ When extrapolated to just half of Canadians with poorly controlled hypertension, that figure expands to an extraordinary $\$ 15.7$ billion in lower costs-a compelling reason to engage pharmacists in the fight against hypertension. Indeed, Hypertension Canada is extremely supportive of expanding the role of pharmacists in hypertension management.

Readers are encouraged to consult the full guidelines in the Canadian Journal of Cardiol$o g y^{2}$ or at www.hypertension.com, where they will find additional professional and patient resources. Pharmacists interested in education relating to hypertension assessment and management can refer to the Hypertension Canada website for education materials, including presentations, videos and documents. 
From the Faculty of Pharmacy and Pharmaceutical Sciences (Lamb), Department of Medicine (Al Hamarneh, Tsuyuki), Faculty of Medicine and Dentistry, University of Alberta, Edmonton, Alberta; the School of Pharmacy (Houle), University of Waterloo, Ontario; and the Department of Medicine (Leung), University of Calgary, Alberta. Contact rtsuyuki@ualberta.ca.

\section{References}

1. Campbell NR, Burgess E, Choi BCK, et al. Methods and overview of the Canadian recommendations. CMAJ 1999;160(9 suppl):S1-S6.

2. Leung AA, Daskalopoulou SS, Dasgupta K, et al. Hypertension Canada's 2017 guidelines for diagnosis, risk assessment, prevention and treatment of hypertension in adults. Can J Cardiol 2017;33(5):557-76.

3. Hua D, Carter S, Bellerive J. Bridging the gap: innovative knowledge translation and the Canadian Hypertension Education Program. Can J Cardiol 2012;8:258-61.

4. Padwal RS, Bienek A, McAlister FA, Campbell NR. Epidemiology of hypertension in Canada: an update. Can J Cardiol 2016;32:687-94.

5. McAlister FA, Wilkins K, Joffres M, et al. Changes in the rates of awareness, treatment and control of hypertension in Canada over the past two decades. CMAJ 2011;183:1007-13. 6. Shiu J, Simpson S, Johnson J, Tsuyuki RT. Quantifying opportunities to affect diabetes management in the community. Can Pharm J (Ott) 2006;139(3):37-8.

7. Chisholm-Burns MA, Kim Lee J, Spivey CA, et al. US pharmacists' effect as a team member on patient care: systematic review and meta-analyses. Med Care 2010;48:923-33. 8. Santschi V, Chiolero A, Burnand A, et al. Impact of pharmacist care in the management of cardiovascular disease risk factors: a systematic review and meta-analysis of randomized trials. Arch Intern Med 2011;171:1441-53.

9. Santschi V, Chiolero A, Paradis G, et al. Pharmacist interventions to improve cardiovascular disease risk factors in diabetes: a systematic review and meta analysis of randomized controlled trials. Diabetes Care 2012;35:2706-17.

10. Santschi V, Chiolero A, Colosimo AL, et al. Improving blood pressure control through pharmacist interventions: a meta-analysis of randomized controlled trials. J Am Heart Assoc 2014;3:e000718.

11. Tsuyuki R, Houle S, Charrois T, et al. A randomized trial of the effect of pharmacist prescribing on improving blood pressure in the community: the Alberta Clinical Trial in Optimizing Hypertension (RxACTION). Circulation 2015;132:93-100. 12. Pojskic N, Jackson M, Malek A, Cimino S. Impact of community pharmacist interventions in hypertension management on patient outcomes: a randomized controlled trial. Can Pharm J (Ott) 2014;147:S17.

13. Tsuyuki RT, Al Hamarneh YN, Jones CA, Hemmelgarn BR. Effectiveness of community pharmacist prescribing and care on cardiovascular risk reduction: randomized controlled RxEACH trial. J Am Coll Cardiol 2016;67(24):2846-54.

14. Houle SKD, Tsuyuki RT, Campbell NRC. The Canadian Hypertension Education Program (CHEP) 2011 guidelines for pharmacists. Can Pharm J (Ott) 2011;144:295-304.
15. Padwal R, Straus S, McAlister F. Cardiovascular risk factors and their effects on the decision to treat hypertension: evidence based review. BMJ 2001;322(7292):977-80.

16. Warwick J, Falaschetti E, Rockwood K, et al. No evidence that frailty modifies the positive impact of antihypertensive treatment in very elderly people: an investigation of the impact of frailty upon treatment effect in the HYpertension in the Very Elderly Trial (HYVET) study, a double-blind, placebo-controlled study of antihypertensives in people with hypertension aged 80 and over. BMC Med 2015;13:78.

17. Williamson JD, Supiano MA, Applegate WB, et al. Intensive vs standard blood pressure control and cardiovascular disease outcomes in adults aged $\geq 75$ years: a randomized clinical trial. JAMA 2016;315:2673-82.

18. Wright JT Jr, Williamson JD, Whelton PK, et al. A randomized trial of intensive versus standard blood-pressure control. N Engl J Med 2015;373:2103-16.

19. Xie X, Atkins E, Lv J, et al. Effects of intensive blood pressure lowering on cardiovascular and renal outcomes: updated systematic review and meta-analysis. Lancet 2016;387:435-43.

20. Olde Engberink RH, Frenkel WJ, van den Bogaard B, Brewster LM, Vogt L, van den Born BJ. Effects of thiazidetype and thiazide-like diuretics on cardiovascular events and mortality: systematic review and meta-analysis. Hypertension 2015;65:1033-40.

21. Roush GC, Ernst ME, Kostis JB, Tandon S, Sica DA. Head-to-head comparisons of hydrochlorothiazide with indapamide and chlorthalidone: antihypertensive and metabolic effects. Hypertension 2015;65:1041-6.

22. Pareek AK, Messerli FH, Chandurkar NB, et al. Efficacy of low-dose chlorthalidone and hydrochlorothiazide as assessed by 24 -h ambulatory blood pressure monitoring. J Am Coll Cardiol 2016;67:379-89.

23. Gradman AH, Parise H, Lefebvre P, et al. Initial combination therapy reduces the risk of cardiovascular events in hypertensive patients: a matched cohort study. Hypertension 2013;61:309-18.

24. Wald DS, Law M, Morris JK, et al. Combination therapy versus monotherapy in reducing blood pressure: metaanalysis on 11,000 participants from 42 trials. Am J Med 2009;122:290-300.

25. Feldman RD, Zou GY, Vandervoort MK, et al. A simplified approach to the treatment of uncomplicated hypertension: a cluster randomized, controlled trial. Hypertension 2009;53:646-53.

26. Jamerson K, Weber MA, Bakris GL, et al. Benazepril plus amlodipine or hydrochlorothiazide for hypertension in high-risk patients. N Engl J Med 2008;359:2417-28. 
27. Lonn EM, Bosch J, Lopez-Jaramillo P, et al. Blood-pressure lowering in intermediate-risk persons without cardiovascular disease. N Engl J Med 2016;374:2009-20.

28. Sherrill B, Halpern M, Khan S, et al. Single-pill vs freeequivalent combination therapies for hypertension: a metaanalysis of health care costs and adherence. J Clin Hypertens (Greenwich) 2011;13:898-909.

29. Law MR, Wald NJ, Morris JK, Jordan RE. Value of low dose combination treatment with blood pressure lowering drugs: analysis of 354 randomised trials. BMJ 2003;326:1427. 30. Corrao G, Nicotra F, Parodi A, et al. Cardiovascular protection by initial and subsequent combination of antihypertensive drugs in daily life practice. Hypertension 2011;58:566-72.

31. Dasgupta K, Quinn RR, Zarnke KB, et al. The 2014 Canadian Hypertension Education Program recommendations for blood pressure measurement, diagnosis, assessment of risk, prevention, and treatment of hypertension. Can J Cardiol 2014;30:485-501.

32. Bangalore S, Messerli FH, Wun CC, et al. J-curve revisited: an analysis of blood pressure and cardiovascular events in the Treating to New Targets (TNT) Trial. Eur Heart $J$ 2010;31:2897-908.

33. Bangalore S, Qin J, Sloan S, et al. What is the optimal blood pressure in patients after acute coronary syndromes? Relationship of blood pressure and cardiovascular events in the PRavastatin OR atorVastatin Evaluation and Infection Therapy-Thrombolysis In Myocardial Infarction (PROVE IT-TIMI) 22 trial. Circulation 2010;122:2142-51.

34. Messerli FH, Mancia G, Conti CR, et al. Dogma disputed: can aggressively lowering blood pressure in hypertensive patients with coronary artery disease be dangerous? Ann Intern Med 2006;144:884-83.

35. Rabkin SW, Shiekh IA, Wood DA. The impact of left ventricular mass on diastolic blood pressure targets for patients with coronary artery disease. Am J Hypertens. 2016;29:1085-93.

36. Rabkin SW. Considerations in understanding the coronary blood flow-left ventricular mass relationship in patients with hypertension. Curr Cardiol Rev. 2017;13:75-83.

37. Anderson CS, Heeley E, Huang Y, et al. Rapid bloodpressure lowering in patients with acute intracerebral hemorrhage. N Engl J Med. 2013;368:2355-65.

38. Qureshi AI, Palesch YY, Barsan WG, et al. Intensive blood-pressure lowering in patients with acute cerebral hemorrhage. N Engl J Med. 2016;375:1033-43.

39. Williams B, MacDonald TM, Morant S, et al. Spironolactone versus placebo, bisoprolol, and doxazosin to determine the optimal treatment for drug-resistant hypertension (PATHWAY-2): a randomised, double-blind, crossover trial. Lancet. 2015;386:2059-68.

40. Calhoun DA, Jones D, Textor S, et al. Resistant hypertension: diagnosis, evaluation, and treatment: a scientific statement from the American Heart Association Professional Education Committee of the Council for High Blood Pressure Research. Circulation 2008;117:e510-26.
41. Myat A, Redwood SR, Qureshi AC, et al. Resistant hypertension. BMJ 2012;345:e7473.

42. Myers MG, Kaczorowski J, Dawes M, Godwin M. Automated office blood pressure measurement in primary care. Can Fam Phys 2014;60:127-32.

43. Daskalopoulou SS, Rabi DM, Zarnke KB, et al. The 2015 Canadian Hypertension Education Program recommendations for blood pressure measurement, diagnosis, assessment of risk, prevention, and treatment of hypertension. Can J Cardiol 2015;31:549-68.

44. Clement DL, De Buyzere ML, De Bacquer DA, et al. Prognostic value of ambulatory blood-pressure recordings in patients with treated hypertension. $N$ Engl J Med 2003;348:2407-15.

45. Lawes CMM, Vander Hoorn S, Rodgers A, for the International Society of Hypertension. Global burden of bloodpressure-related disease, 2001. Lancet 2008;371:1513-8.

46. Forouzanfar MF, Liu P, Roth GA, et al. Global burden of hypertension and systolic blood pressure of at least 110 to 115 mm hg, 1990-2015. JAMA 2017;317(2):165-82.

47. Gee ME, Bienek A, McAlister FA, et al. Factors associated with lack of awareness and uncontrolled high blood pressure among Canadian adults with hypertension. Can J Cardiol 2012;28:375-82.

48. Grover SA, Lowensteyn I, Joseph L, et al. Patient knowledge of coronary risk profile improves the effectiveness of dyslipidemia therapy: the CHECK-UP study: a randomized controlled trial. Arch Intern Med 2007;167:2296-303.

49. Benner JS, Erhardt L, Flammer M, et al. A novel programme to evaluate and communicate 10 -year risk of CHD reduces predicted risk and improves patients' modifiable risk factor profile. Int J Clin Pract 2008;62:1484-98.

50. D’Agostino RB, Vasan RS, Pencina MJ, et al. General cardiovascular risk profile for use in primary care: the Framingham Heart Study. Circulation 2007;117:743-53.

51. Soureti A, Hurling R, Murray P, et al. Evaluation of a cardiovascular disease risk assessment tool for the promotion of healthier lifestyles. J Cardiovasc Prev Rehabil 2010;17:519-23. 52. Grover SA, Lowensteyn I, Joseph L, et al. Patient knowledge of coronary risk profile improves the effectiveness of dyslipidemia therapy. Arch Intern Med 2007;167:2296-30.

53. Teo KK, Ounpuu S, Hawken S, et al. Tobacco use and risk of myocardial infarction in 52 countries in the INTERHEART study: a case-control study. Lancet 2006;368:647-58. 54. Saba M, Diep J, Saini B, Dhippayom T. Meta-analysis of the effectiveness of smoking cessation interventions in community pharmacy. J Clin Pharm Ther 2014;39:240-7.

55. Stead LF, Hartmann-Boyce J, Perera R, Lancaster T. Telephone counselling for smoking cessation. Cochrane Database Syst Rev 2013;(8):CD002850.

56. Cahill $\mathrm{K}$, Lancaster $\mathrm{T}$. Workplace interventions for smoking cessation. Cochrane Database Syst Rev 2014;(4): CD003440.

57. Abegaz TM, Shehab A, Gebreyohannes EA, et al. Nonadherence to antihypertensive drugs: a systematic review and meta-analysis. Medicine Open 2017;96(4):e5641-9. 
58. Chockalingam A, Bacher M, Campbell N, et al. Adherence to management of high blood pressure: recommendations of the Canadian Coalition for High Blood Pressure Prevention and Control. Can J Public Health 1998;89(Suppl 2):15-6.

59. Fodor JG, Cutler H, Irvine J, et al. Adherence to nonpharmacological therapy for hypertension: problems and solutions. Can J Public Health 1998;89(Suppl 2):112-5.

60. Vrijens B, Vincze G, Kristanto P, Urquhart J, Burnier M. Adherence to prescribed antihypertensive drug treatments: longitudinal study of electronically compiled dosing histories. BMJ 2008;336(7653):1114-7.

61. Faulkner MA, Wadibia EC, Lucas BD, Hilleman DE. Impact of pharmacy counseling on compliance and effectiveness of combination lipid-lowering therapy in patients undergoing coronary artery revascularization: a randomized, controlled trial. Pharmacotherapy 2000;20:410-6.

62. Marra C, Johnston K, Santschi V, et al. Cost-effectiveness of pharmacist care for managing hypertension in Canada. Can Pharm J (Ott) 2017;150(3):184-97. 